\title{
Assessment of the impact of TS assimilation from ARGO floats in the Mediterranean Sea
}

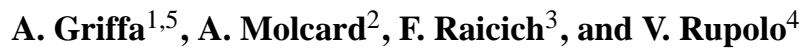 \\ ${ }^{1} \mathrm{CNR}$, ISMAR/La Spezia, Italy \\ ${ }^{2}$ LSEET, Univ. Toulon, France \\ ${ }^{3} \mathrm{CNR}$, ISMAR/Trieste, Italy \\ ${ }^{4}$ ENEA, Casaccia, Roma, Italy \\ ${ }^{5}$ RSMAS, University of Miami, FL, USA
}

Received: 2 May 2006 - Published in Ocean Sci. Discuss.: 3 June 2006

Revised: 6 November 2006 - Accepted: 23 November 2006 - Published: 5 December 2006

\begin{abstract}
In this paper, the impact of assimilating Temperature $(\mathrm{T})$ and Salinity $(\mathrm{S})$ profiles from Argo floats in the Mediterranean Sea is quantitatively investigated using the Observing System Simulation Experiments (OSSE) approach. The impact of varying the number of floats and their launch positions is considered, using numerical simulations with a MOM model and a reduced-order multivariate Optimal Interpolation scheme (SOFA) for assimilation. Realistic float coverage and launch positions used during the first MFSTEP phase are considered, as well as "ideal" density coverage that can be envisioned for the future, corresponding to a double coverage with respect to MFSTEP and with floats released along the VOS tracks. The most effective float trajectories are identified, showing that frontal regions play a major role, and that it is crucial to maintain a sufficient coverage of them. In addition to this, a comparison is also performed between the results obtained from MEDARGO floats in ideal conditions and results from "ideal" profiles taken at fixed points along the VOS tracks, as for the XBT data. For consistency the coverage considered is double the actual XBT coverage during MFSTEP, resulting in a threefold increase in the number of profiles compared to the MEDARGO experiment. The maximum error reduction is of approximately $10 \%$, suggesting that spatially coarser profiles from floats can be more efficient since they follow flow features.
\end{abstract}

\section{Introduction}

The main objective of the MFSTEP (Mediterranean Forecasting System Towards Environmental Predictions) Project is the development of an operational forecasting system for the Mediterranean Sea, based upon a Near Real Time (NRT) observing system and a numerical model forecasting system.

Correspondence to: A. Griffa

(agriffa@rsmas.miami.edu)
The data provided by the observing system are assimilated in the numerical models, allowing for the forecasting capabilities. In this framework, understanding the impact of different measuring platforms in terms of sampling schemes and assimilation methods is of crucial importance. The Observing System Simulation Experiments (OSSE) (Arnold and Day, 1986; Kindle, 1986) play an important role in assessing and comparing the usefulness of the various platforms, sampling strategies and data combinations which can be adopted in the observing system.

As part of the MFSTEP project, NRT acquisition and processing of temperature $\mathrm{T}$ and salinity $\mathrm{S}$ profiles provided by autonomous profiling floats have been tested and are presently operationally implemented. The floats, referred to as MEDARGO, are part of the global ocean Argo project (Poulain, 2005; http://www.argo.ucsd.edu) which plays a major role in the global ocean observing system. Argo aims to provide a world-wide continuous monitoring of the temperature, salinity, and velocity of the upper ocean, with all data being released and made publicly available within hours after collection. Argo floats are programmed to freely drift at a given nominal depth for a time interval of the order of days, then resurfacing to communicate via satellite information on TS profiles and on positions. In particular, for MEDARGO floats the repetitive cycle is performed over a period of approximately 5 days (Fig. 1): the floats descend to the prescribed neutral depth of $350 \mathrm{~m}$, drift at that level and then dive down to about $700 \mathrm{~m}$ before ascending to the surface while collecting TS data. During their short period at the surface, the profilers are located by, and the data are telemetered to, the satellite Argos system. The nominal depth of $350 \mathrm{~m}$ is chosen to maximize information on the very important LIW (Levantine Intermediate Water) water mass, whose core is approximately located at that depth.

TS profile information from Argo floats are presently assimilated in a number of operational systems (http: //www.argo.ucsd.edu/FrUse_by_Operational.html). In the

Published by Copernicus GmbH on behalf of the European Geosciences Union. 


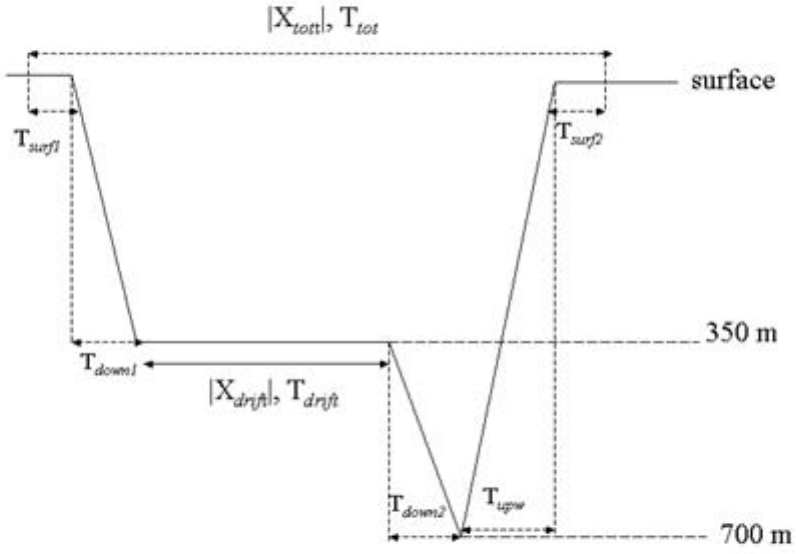

Fig. 1. Schematic of the MEDARGO float cycle.

Mediterranean Sea they provide, together with the XBT data collected along the Volunteer Observing Ship (VOS) tracks (Manzella et al., 2003), the backbone of the data assimilation and forecast system at basin scale (Demirov et al., 2003). Since the Mediterranean Sea has a particularly strong salinity contribution to density, the combined observation of temperature and salinity profiles is expected to be of particular relevance.

The objective of the present work is to quantitatively investigate the impact of assimilating TS from MEDARGO floats in the Mediterranean Sea, and to identify which density coverage and launch positions appear particularly effective. As such, the work is intended to contribute to future planning of sampling strategies for the Mediterranean Sea. The OSSE approach is followed, where the "true" ocean state is assumed known and represented by a reference simulation from which synthetic data are extracted. The advantage of this approach is that it allows a quantitative assessment of the data assimilation since the truth is known. The disadvantage is that synthetic data are used which are completely compatible with the models so that the results might be optimistic with respect to assimilation of real in-situ data. Despite this limitation, OSSEs provide a very useful tool and they have been widely used to assess the quality of observing systems in the atmosphere (e.g. Arnold and Dey, 1986; Rohaly and Krishnamurti, 1993) and in the ocean (Kindle, 1986; Bennett, 1990; Barth and Wunsch, 1990; Hernandez et al., 1994; Hackert et al., 1998; Raicich and Rampazzo, 2003; She et al., 2006). The present application builds on a previous work performed in the Mediterranean Sea in the framework of the MFSPP (Mediterranean Forecasting System Pilot Project, Raicich and Rampazzo, 2003) focused on assessing the impact of assimilating XBT data. The same methodology as in Raicich and Rampazzo (2003) is used. The Mediterranean GCM used as a basis for the simulations is a version of the Geophysical Fluid Dynamics Laboratory MOM-1 (Pacanowski et al., 1990), having $1 / 8^{\circ} \times 1 / 8^{\circ}$ hori- zontal resolution and 31 vertical levels, i.e. the same model operationally used in MFSPP and in the first year of MFSTEP. The assimilation system is the same as presently used in MFSTEP, based on the reduced-order multivariate Optimal Interpolation scheme SOFA (System for Ocean Forecasting and Analysis, De Mey, 1994, 1997; De Mey and Benkiran, 2002).

In order to focus on the impact of TS profiles from MEDARGO floats, their assimilation is considered in isolation, i.e. without the contribution of other data platforms present in the Mediterranean Sea. The OSSE experiments focus on the uncertainty in the initial ocean state and study the impact of varying the number of floats and their launch positions, using a bivariate data assimilation for TS. Realistic launch positions used during the first MFSTEP phase are considered, as well as "ideal" positions that can be envisioned for the future, along the VOS tracks. The most effective float trajectories are identified, and related to the dynamical characteristics of the flow. In addition to this, also a qualitative comparison is performed between the results obtained from MEDARGO floats in ideal conditions and results from "ideal" profiles taken along the VOS tracks, as for the XBT data.

The paper is organized as follows. The general methodology used in this work is described in Sect. 2, while in Sect. 3 the specific characteristics of the experiments are provided. The experiment results are reported in Sect. 4, and a brief summary and conclusions are given in Sect. 5 .

\section{Methodology}

The OSSE methodology used here is based on the identical twin experiment approach, which uses synthetic numerically simulated data instead of real in-situ ones for the assimilation task. Details on the MOM-1 Mediterranean GCM used for the simulations can be found in Korres et al. (2000) and Demirov et al. (2003). The model is forced by ECMWF 6hourly operational analyses of $10 \mathrm{~m}$-wind and $2 \mathrm{~m}$-air temperature, used also in the surface heat flux calculation. Surface salinity is relaxed to the MED6 climatology (Brasseur et al, 1996; Fichaut et al., 1998).

Three main simulations are performed: the Control run, which represents the "true ocean" state, and from which the synthetic data are extracted; the Assimilation run, which is initialized from different initial conditions from the Control run, to represent our incomplete knowledge of the true state of the ocean, and in which the data from the Control are assimilated; the Free run, which is initialized as the Assimilation run but without data assimilation. The latter provides a reference simulation, where the "wrong" initial conditions evolve according to forcing and dynamics. All runs are driven by the same external forcing.

Synthetic floats are launched in the Control and advected by the numerical velocity field, using parameters in the 
same range as for real in-situ MEDARGO floats. The Lagrangian integration is performed using the off-line Ariane algorithm (Blanke et al., 1999) modified in order to simulate the MEDARGO cycle (Fig. 1). Floats drift at the prescribed $350 \mathrm{~m}$ depth for a time period $\mathrm{T}_{\text {drift }} \sim 5.3$ days, and then dive down to $700 \mathrm{~m}$ with a vertical downward velocity of $5 \mathrm{~cm} / \mathrm{s}$, and ascend to the surface with an upward velocity of $10 \mathrm{~cm} / \mathrm{s}$, while collecting TS data. They then spend $6 \mathrm{~h}$ drifting at the surface, before descending to $350 \mathrm{~m}$ and repeating the cycle. Salinity and temperature profiles are provided at each cycle at the location of the floats and these profiles, extracted from the Control run, are considered as our real data and assimilated in the Assimilation run.

Data assimilation is performed by means of SOFA on a sequence of 5-day assimilation cycles. The SOFA order reduction is achieved by projecting the state vector onto vertical EOFs, that represent the eigenvectors of the error covariance matrix for the forecast. The TS bivariate EOFs are computed from a 1993-1999 GCM run according to Sparnocchia et al. (2003). The data error covariance radius is $10^{-6} \mathrm{~km}$ and the e-folding time is $10^{-6}$ days, implying that the data errors are uncorrelated with each other. The forecast error covariance radius is $45 \mathrm{~km}$ and e-folding time is 105 days, which results in giving all the data within the assimilation window practically the same weight. These parameters were chosen to be the same as in the MFSTEP operational code.

The success of the assimilation is quantified in terms of the convergence of the Assimilation run towards the Control. In other words, a successful data assimilation is expected to correct the "wrong" initial conditions, driving the ocean state toward the "truth". The convergence of the Assimilation run towards the Control run is assessed by means of standard deviations of differences (of the concerned variables) between the two runs (Miyakoda et al., 1969). Standard deviations, which are computed using the anomalies relative to the spatial means, are adopted instead of root-mean-squared differences, computed with the full fields, since they are more sensitive to data assimilation (Raicich and Rampazzo, 2003). In order to uniform the performance errors when comparing different simulations, the standard deviations of the Control minus Assimilation is normalized by the corresponding standard deviation for the Free run, used for reference since it shows the ability of the model to converge toward the Control due to the atmospheric forcing only.

Standard deviations are computed for the western Mediterranean (Alboran Sea, Algerian Current region, Gulf of Lions, Tyrrhenian Sea and northern Sicily Channel) and eastern Mediterranean (southern Sicily Channel, Adriatic Sea, Ionian Sea, Aegean Sea and Levantine Basin), and in three layers, defined as follows: L1, which includes 10 model levels from 5 to $240 \mathrm{~m}$ depth roughly corresponding to the surface waters; L2, including 4 levels from 280 to $400 \mathrm{~m}$ approximately corresponding to the Levantine Intermediate Water (LIW) layer; L3, including 17 levels from $440 \mathrm{~m}$ to the sea bottom.

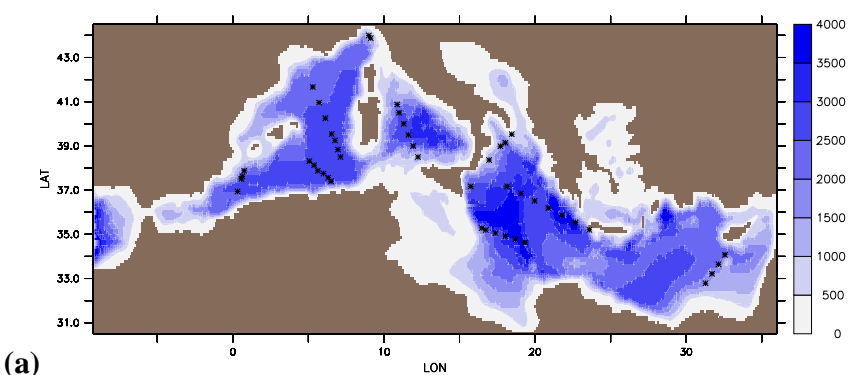

(a)

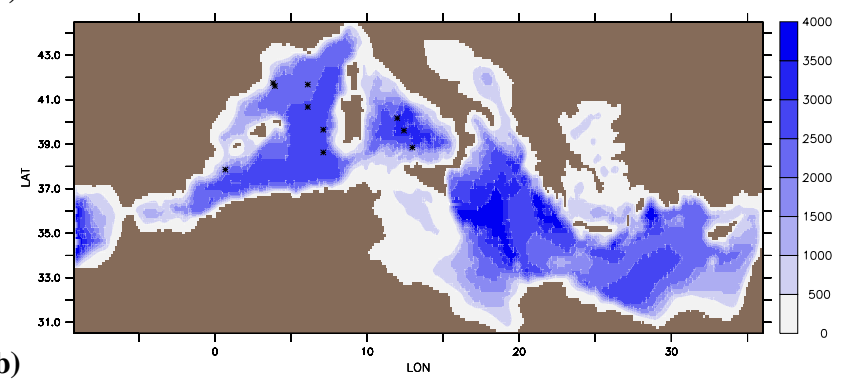

Fig. 2. Launching sites for numerical MEDARGO floats in experiments: 47_WINTER, 47_SUMMER in upper panel; 10_SUMMER in lower panel. Blue shades indicate bottom depth in meters.

\section{OSSE experiments}

The OSSE configuration has been first tested in a number of preliminary experiments. Three main experiments have then been performed and they are reported here.

In the first two experiments, 47_SUMMER and 47_WINTER, an "idealized" situation with respect to the actual launchings during MFSTEP is considered. A total of 47 floats are launched, which is approximately double the total number of MEDARGO floats launched during MFSTEP (about 20). It can therefore be considered as a realistic upper limit for a sampling strategy in the Mediterranean Sea. The floats are launched along VOS tracks, considering a summer and a winter situation respectively. The launching sites (Fig. 2a) are evenly spaced along the VOS tracks where the depth is greater than $700 \mathrm{~m}$. VOS tracks are envisioned to be used in the future for operational MEDARGO launchings.

In the third experiment, 10_SUMMER, 10 floats are launched, considering the same launching sites (Fig. 2b) as for the in-situ MFSTEP MEDARGO floats deployed between June and October 2004. Notice that these initial test launches, performed mostly from research vessels, are all situated in the Western Mediterranean Sea, so that the coverage there is approximately half that for the 47_SUMMER experiment (while of course there is no coverage in the eastern basin). This experiment will provide insights on the assimilation impact of the initial MFSTEP sampling at least in the Western Mediterranean.

The two seasonal configurations SUMMER and WINTER are defined according to the initial state of the ocean, and 

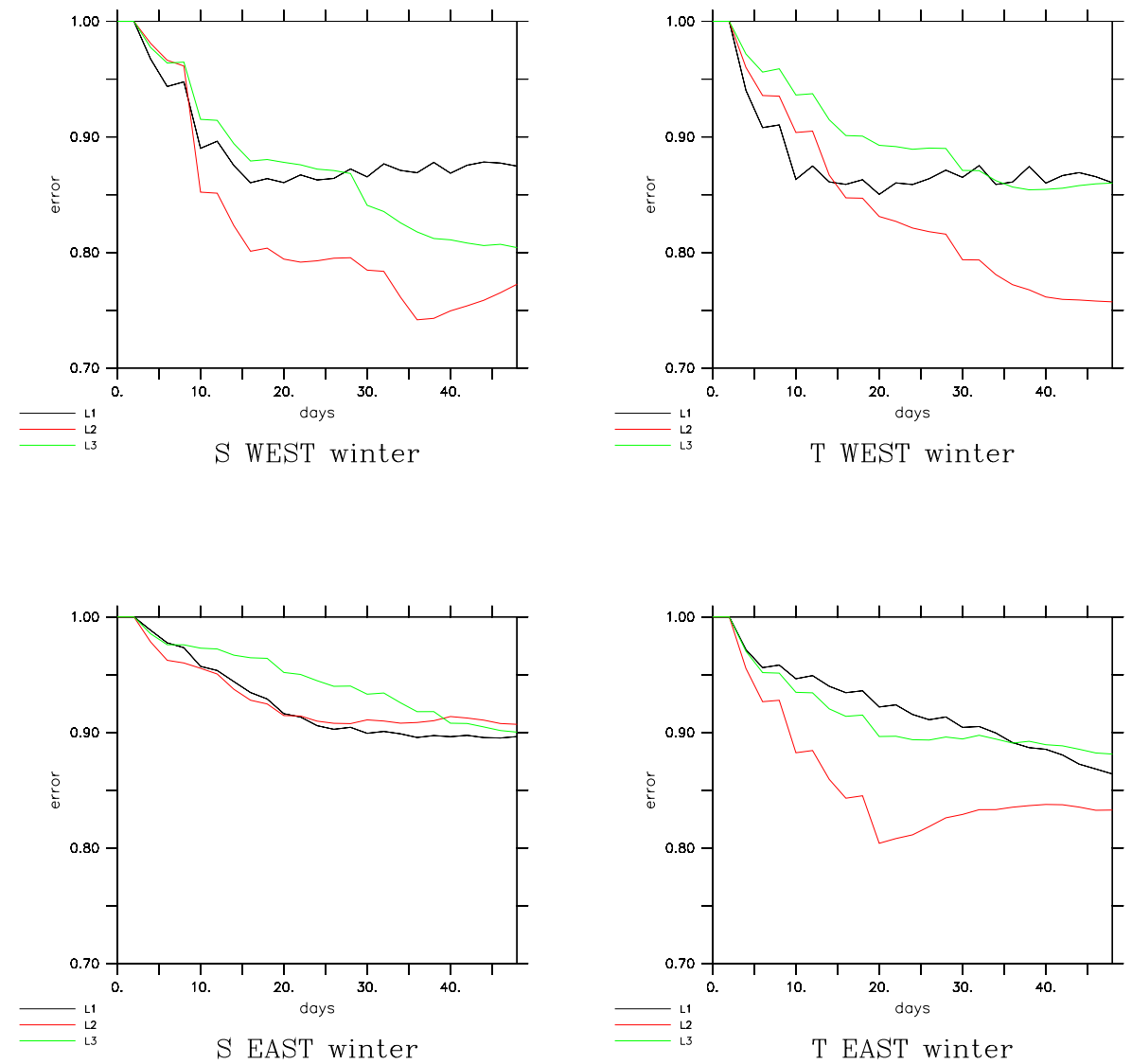

Fig. 3. Relative error versus time in days for experiment 47_WINTER. Upper panels are relative to the Western basin and lower panels to the Eastern basin; Right panels are relative to temperature $\mathrm{T}$ errors and left panels to salinity $\mathrm{S}$ errors. The three lines in each panel indicate the layers: L1 in black, L2 in red and L3 in green.

each experiment lasts for 60 days, corresponding to 12 assimilation cycles. The summer OSSE Controls are initialized on 1 September 1999, while the winter OSSE controls are initialized in 1 February 2000. Free and Assimilation runs are initialized on 1 September 1998 for the summer and on 1 February 1999 for winter. All the initialization fields are taken from an interannual simulation with data assimilation forced by ECMWF 6-hourly analyses.

The results of the idealized experiments with 47 floats are compared with results of "idealized VOS" experiments, obtained by assimilating profiles at fixed points along the VOS tracks with a weekly total coverage approximately double with respect to the maximum weekly XBT coverage obtained in MFSTEP (Raicich, 2006). The comparison provides comparative information on different data platforms and data coverage and sampling.

\section{Results}

\subsection{Idealized sampling experiments}

Results obtained for the 47_WINTER and 47_SUMMER experiments are summarized in terms of relative errors versus time in Figs. 3 and 4, respectively. The relative error is the ratio between the Assimilation run error (AssimilationControl) and the Free run error (Free-Control), and it quantifies the improvement over the Free run due to the data assimilation.

Figures 3 and 4 show the errors for $\mathrm{T}$ and $\mathrm{S}$ for both the Western and Eastern basins and for the three layers L1, L2 and L3. As it can be seen, the assimilation is successful in all cases, providing a decrease in the assimilation error, especially marked during the first two assimilation cycles. Only in some cases, as for the S error in the winter in the Eastern basin, the initial decrease is not very stong, probably because the floats do not sample strong salinity gradients at initial times. Final assimilation errors reach minimum values of $75 \%$, indicating an error reduction of $20-30 \%$.

During winter, the best results are obtained for the 

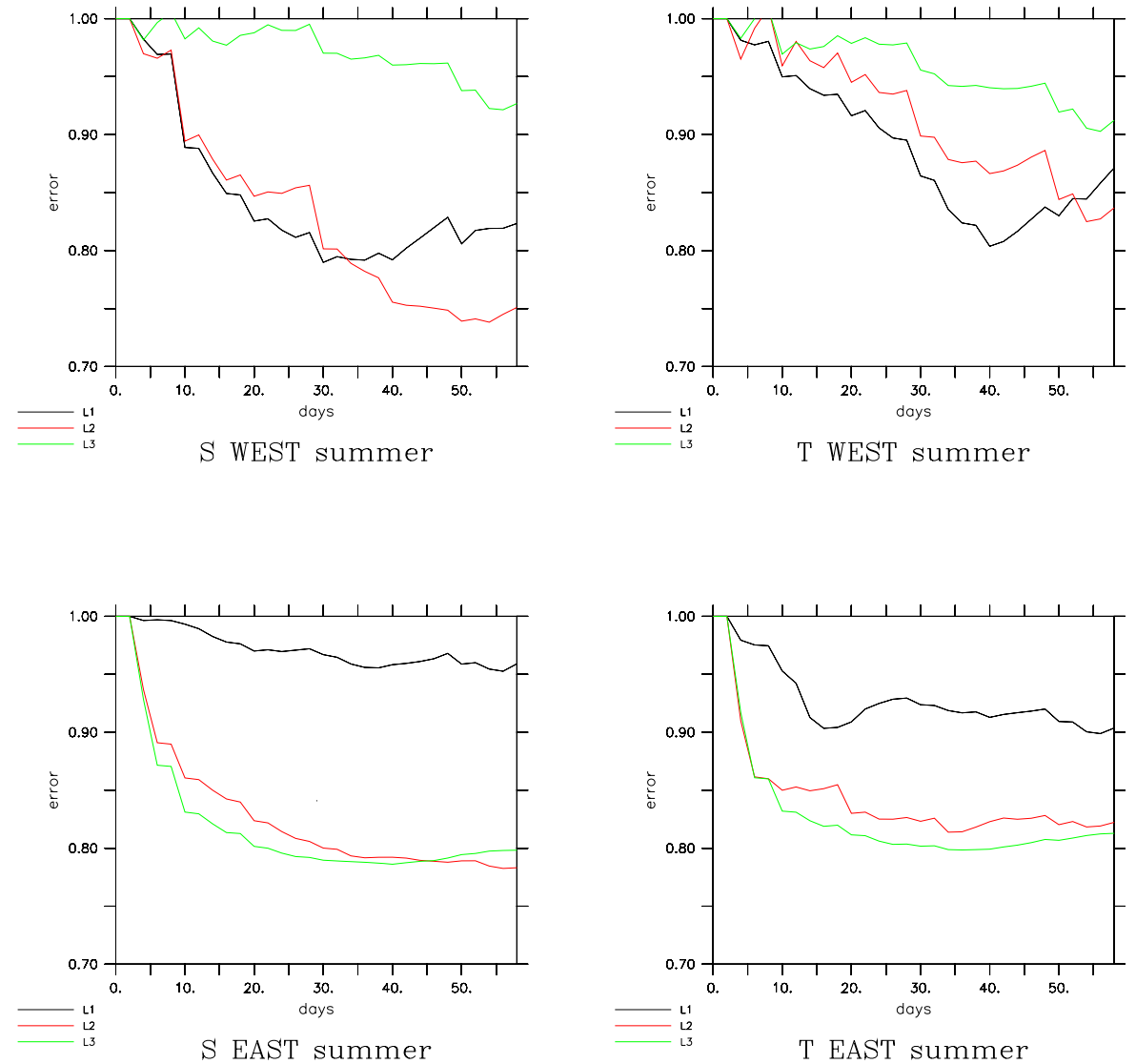

Fig. 4. Relative error versus time in days for experiment 47_SUMMER. Upper panels are relative to the Western basin and lower panels to the Eastern basin; Right panels are relative to temperature $\mathrm{T}$ errors and left panels to salinity $\mathrm{S}$ errors. The three lines in each panel indicate the layers: L1 in black, L2 in red and L3 in green.

intermediate layer L2, and also in summer the L2 correction is always significant. This is probably due to the fact that the temperature and salinity gradients connected with the Levantine Intermediate Water (LIW) fronts are well marked in all seasons. We will come back on this point in the following, when analyzing specific examples of $\mathrm{T}$ and $\mathrm{S}$ fields. L1 corrections are not always as effective as for L2, possibly because of the higher temporal and spatial variability of the surface fronts.

An important question to be addressed is which trajectories contribute the most to the error reduction. In order to illustrate this point, we consider a specific case of salinity $\mathrm{S}$ assimilation for 47_WINTER, that can be considered as "typical". Maps of S for the Control and the Free runs are shown in Figs. 5a, b at $400 \mathrm{~m}$ (i.e. at the basis of the second level L2) and at $\mathrm{t}=35$ days, i.e. after that the errors (Fig. 3) have reached an approximately steady level. The difference between the two fields (illustrated by the misfit in Fig. 6a) is due to the persistent effect of the different initial conditions of the two runs, and it is especially evident in correspondence to the main fronts between water masses. A front between the LIW and the less salty water of Adriatic origin is clearly visible South of the Otranto Strait in the Ionian Sea. In the Free run the penetration of Adriatic water is more enhanced than in the Control. Another strong front is present West of the Sardinia Channel, dividing the western water from the more saline eastern water influenced by the LIW advection. The different mesoscale structure of the front in the Control and in the Free runs determines a strong misfit in this area. Finally, a third area of strong misfit can be noticed close to the Northwestern coast. This is probably due to an upwelling event which is not correctly represented by the Free run.

The float trajectories are shown in Fig. 6a superimposed on the (Control-Free) misfit. As it can be seen the two main fronts in the Ionian Sea and in the Sardinia Channel are well sampled, and also the misfit area in the North West is sampled by one trajectory. We expect that these trajectories, sampling the areas of maximum misfit, will be the ones that will contribute the most to the assimilation.

In order to confirm this hypothesis, we compare the $\mathrm{S}$ distribution for the Assimilation and Free runs at the same depth and time as for the Control (Figs. 5c, b). The differences between Assimilation and Free are expected to be indicative of the effect of the assimilation, correcting the "wrong" initial 


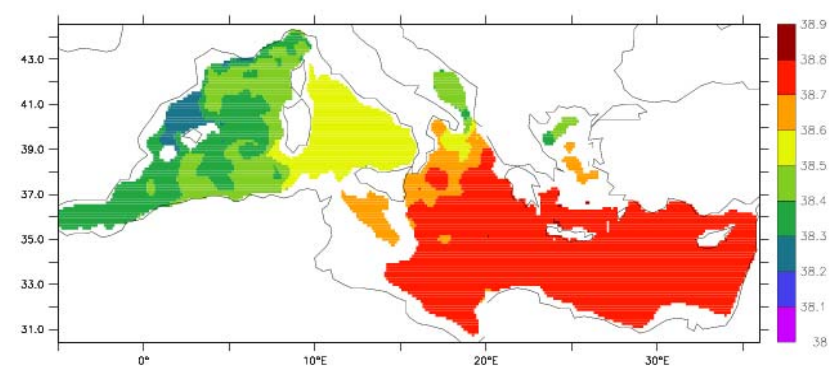

(a)

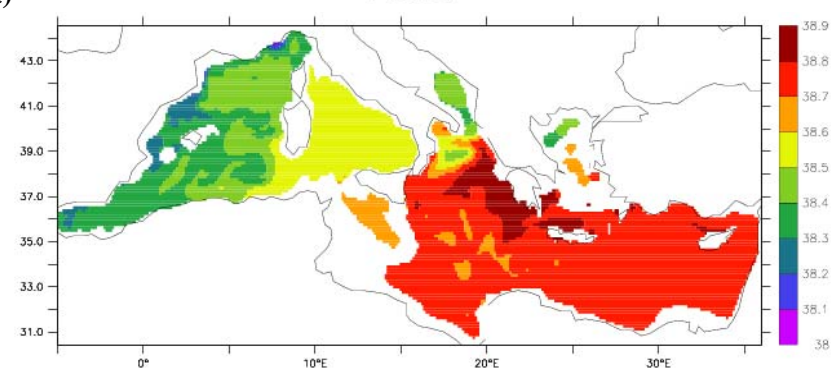

(b)

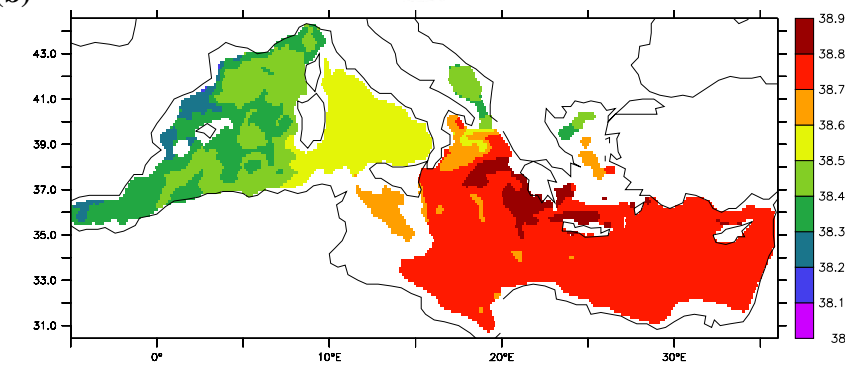

(c)

assim

Fig. 5. Salinity maps for experiment 47 WINTER at $400 \mathrm{~m}$. and at time $\mathrm{t}=35 \mathrm{~d}$. Upper panel corresponds to the Control run, middle panel to the Free and lower panel to the Assimilation.

conditions. The maximum misfit values for (AssimilationFree) (Fig. 6b) occur indeed in the same areas as for (ControlFree) (Fig. 6a) and they have the same sign, indicating that the assimilation is correcting the initial conditions and causing a convergence toward the Control. Superposition of the trajectories to the misfit (Fig. 6b) clearly show that the main assimilation impact is due to the trajectories in the frontal regions, as hypothesized above, while trajectories in the other regions contribute only marginally to the error reduction.

The VOS tracks from which floats with trajectories along fronts have been launched are mainly two (Fig. 2): the track in the Ionian Sea (track 5, see Fig. 7) and the track across the Algerian basin (track 2, see Fig. 7). In the specific example of Fig. 5, also the first trajectory of the track in the Liguro/Tyrrhenian Sea (track 4) contributes significantly, but this is more episodic and related to an upwelling event rather than to a permanent water mass front. We notice, that while launching positions on specific tracks influence the assimi-

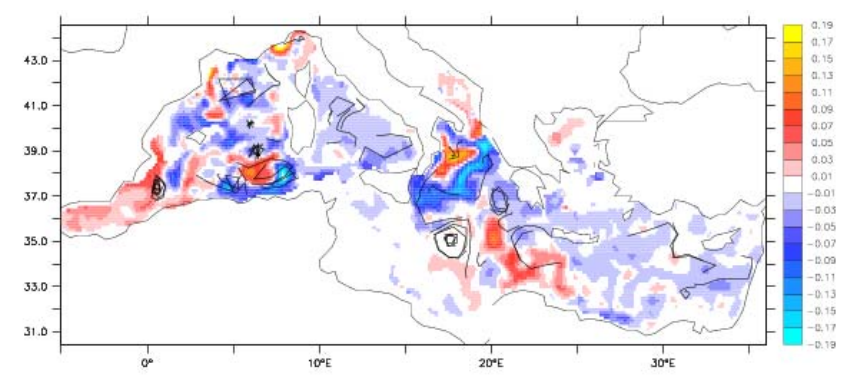

(a)

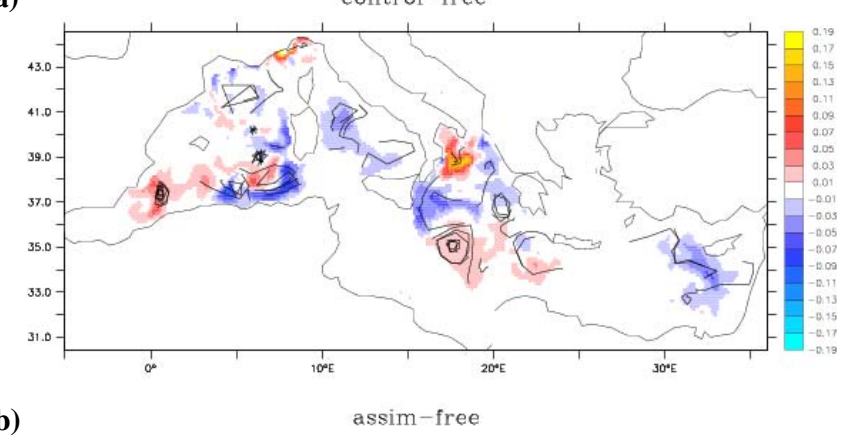

Fig. 6. Trajectories (computed over the whole integration period) superimposed to salinity misfit maps for experiment 47_WINTER at $400 \mathrm{~m}$. and at time $\mathrm{t}=35 \mathrm{~d}$. Upper panel corresponds to the misfit (Control-Free) and lower panel to (Assimilation-Free).

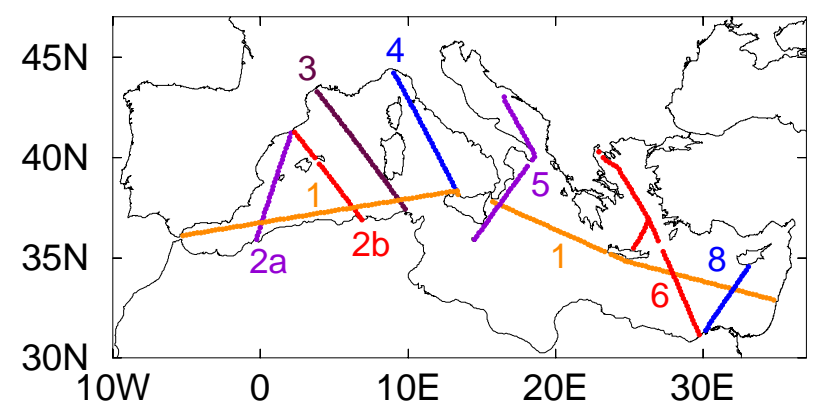

Fig. 7. VOS track coverage.

lation results at the relative short time scales of 1-2 months considered here, Argo floats are expected to live for years, so that the launch positions are not expected to be very important at later time as there is no control on the long term trajectories.

Results for temperature maps (not shown) are qualitatively similar. This is not surprising, given that the main fronts of $\mathrm{T}$ and $\mathrm{S}$ approximately coincide at intermediate depth, given that the LIW is characterized by salty and warm water. Also for the summer experiment, 47_SUMMER, at intermediate depth the situation is qualitatively similar, since the fronts are persistent in both seasons.

The surface level, L1, results (not shown) are qualitatively 

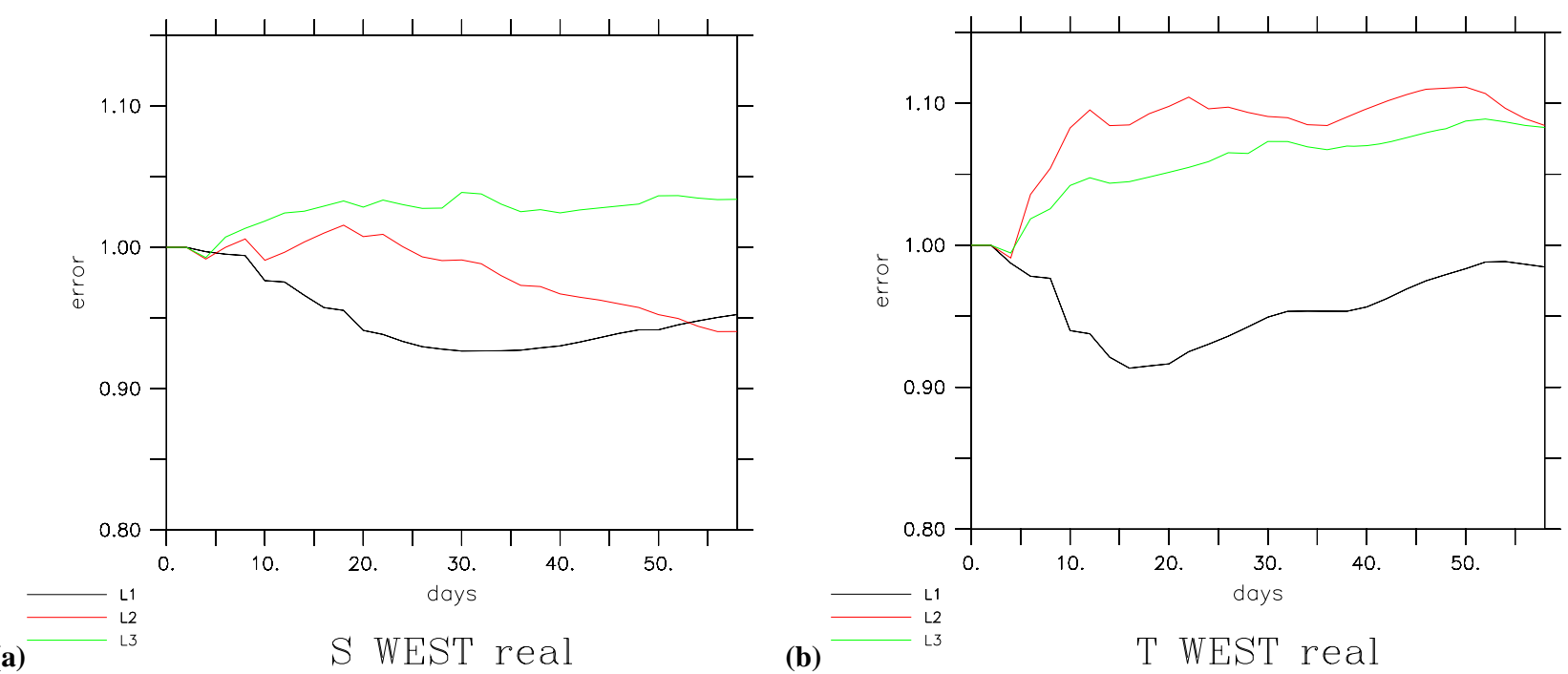

Fig. 8. Relative error versus time in days for experiment 10_SUMMER. The panels are relative to the Western basin. Right panel is relative to temperature T errors and left panel to salinity S errors. The three lines in each panel indicate the layers: L1 in black, L2 in red and L3 in green.

similar to L2 in the Western basin, where the main gradients of T and S occur West of the Sardinia Channel. As for L2, a well defined front exists between the fresher and cooler water of Atlantic origin and the saltier and warmer Mediterranean water. This leads to a consistent correction of $\mathrm{T}$ and $\mathrm{S}$ in $\mathrm{L} 1$ during both winter (Fig. 3) and summer (Fig. 4). In the Eastern basin, instead, the persistent gradient with the Adriatic water which characterizes L2 is not present in L1. The main gradients and the more marked differences between Control and Free occur in the Levantine basin and, especially for $\mathrm{T}$ in summer, close to the coast. These fronts are not as persistent and well defined as in L2, and they are not consistently sampled from trajectories from a given track. Also, the fronts are not necessarily occurring simultaneously in both $\mathrm{T}$ and $\mathrm{S}$. As a consequence, the assimilation in L1 does not appear as effective as in L2, especially in summer. The VOS tracks that correspond to the most effective trajectories are track 2 in the Western basin, as for L2, and track 1 in the Eastern basin.

\subsection{Real sampling experiment}

The results of 10_SUMMER are summarized in Fig. 8 in terms of relative errors for $\mathrm{T}$ and $\mathrm{S}$ versus time. Only the Western basin statistics are reported given that all the floats have been launched there (Fig. $2 b$ ).

The results show that the assimilation correction is not as effective with this reduced sampling. The $\mathrm{S}$ errors remain greater than $90 \%$, while the $\mathrm{T}$ errors show a temporary increase, especially at intermediate level L2. In order to understand the reasons for this increase, we compare the maps of the misfits (Control-Free) and (Assimilation-Free) (Fig. 9), computed for $\mathrm{T}$ at $400 \mathrm{~m}$. The scales are chosen to be the same for both misfits, to facilitate the comparison, and white regions in Fig. 9 indicate areas off scale, i.e. with greater values. The trajectories are superimposed on the (Assimilation-Free) map (Fig. 9). Notice the presence of a negative eddy anomaly South-West of Sardinia in (ControlFree) (Fig. 9), which corresponds to a positive eddy anomaly in (Assimilation-Free) (Fig. 9). This indicates that the assimilation tends to correct in the opposite direction with respect to the Control, therefore increasing the error instead of reducing it. This "wrong" positive anomaly in (Assimilation-Free) is likely to be due to the propagation of an initially correct anomaly due to the trajectory launched West of the tip of Sardinia (Fig. 2b). This trajectory in fact samples regions of positive anomaly in (Control-Free), situated just North East of the negative eddie. The propagation of the correction, combined with the fact that the negative eddy is not sampled by any trajectory, causes the "wrong" correction of the assimilation. This does not happen in the surface layer L1 (not shown), probably because the advection is different in L1.

In conclusion, the results of experiment 10_SUMMER indicates that the assimilation is not as effective for reduced sampling, especially in the frontal zones, and it can lead to spurious corrections and possible temporary error increases.

\subsection{Comparison with idealized VOS experiments}

It has been shown in Sect. 4.1 that with the idealized sampling, corresponding to approximately twice the actual deployment strategy during MFSTEP, the TS assimilation from the MEDARGO floats can significantly improve the forecast. In order to complete the assessment, these results are 


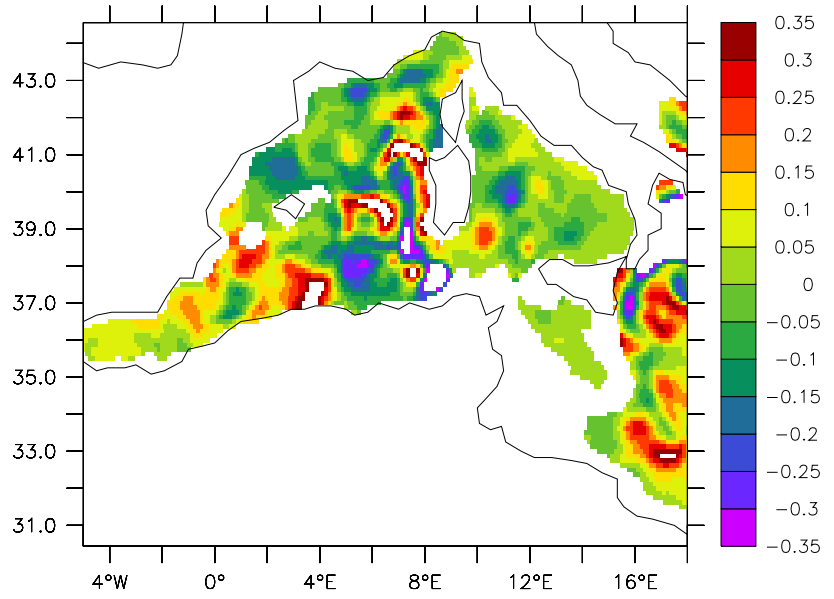

(a)

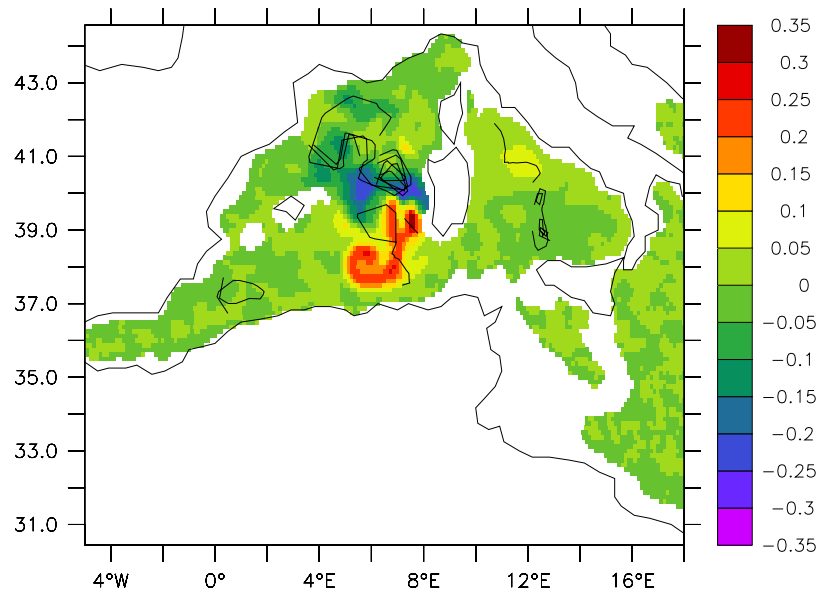

(b)

$$
\text { assim-free }
$$

Fig. 9. Misfit temperature T maps for experiment 10_SUMMER at $400 \mathrm{~m}$ and at time $\mathrm{t}=35 \mathrm{~d}$. Upper panel corresponds to the misfit (Control-Free) and lower panel to (Assimilation-Free) Trajectories (computed over the whole integration period) are superimposed on the (Assimilation-Free) misfit.

compared with assimilation from profiles along the VOS tracks, as for the XBTs data (Manzella et al., 2003). The goal is to compare two different types of sampling scheme, one with profiles taken at fixed points along the VOS tracks (as for XBT), and the other with profiles taken along float trajectories (as for MEDARGO).

For the sake of comparison, both Temperature and Salinity are assimilated also for VOS profiles, as if expendable CTDs (XCTDs) were available instead of XBT. Also for consistency, the "idealized VOS" sampling is chosen to have a weekly coverage corresponding to approximately twice the present maximum weekly MFS sampling. This is achieved maintaining the same spatial design as in the MFS, based upon the tracks in Fig. 7 sampled every 12 nautical miles with a maximum depth profile of $770 \mathrm{~m}$, while the time coverage is assumed regular and more frequent than in reality. Track 1 is covered once every two weeks, tracks $2 \mathrm{a}$ and $7 \mathrm{a}$ only in the odd weeks and tracks $2 b$ and $7 b$ only in the even weeks. The other tracks are covered weekly. The resulting total number of profiles available per week is approximately 200 both in the western and the eastern basin, and it is approximately twice the maximum weekly number of observations performed in the Mediterranean Sea in 2004 and 2005 (Raicich, 2006). By comparison, the idealized MEDARGO experiments are characterized by a significantly smaller number of profiles (approximately 65 per week over the whole Mediterranean Sea).

The assimilation of VOS data and MEDARGO data is performed using the same method (Sect. 2), except for the length of the assimilation cycle, namely 7 days for VOS data and 5 days for MEDARGO. The reason for such difference is that VOS typically repeat the same track with a periodicity multiple of one week. Details can be found in Raicich (2006).

Figure 10 depicts the relative error computed with the two assimilation strategies, for the winter period in the three layers. Analogous results are obtained for the summer (not shown). For simplicity, we focus on the Temperature error in the western basin (left panels) and in the eastern basin (right panels). At first glance, the relative error plots show for all experiments, in all layers, an improvement of the forecast. The error reduction with floats and VOS profiles are consistent and generally differ by less than $10 \%$.

In the surface layer, L1, VOS data turn out to be more effective than MEDARGO. This situation is found also in L2 but only in the Western Basin (left panel), while in the Eastern Basin (left panel) the assimilation of both data sets produces essentially the same error reduction after two weeks. In L3 the two data sets are almost equally effective. The lower relative error, T obtained with VOS data in L1 in both basins and L2 in the West Mediterranean, appears to be mostly determined by the assimilation of relatively large profile amounts during first two weeks in the West basin and the first week in the East basin. After the initial abrupt error reductions, the improvement with MEDARGO and VOS data do not differ much.

In the Western basin (left panels), MEDARGO floats continuously correct the forecast when time is increasing, as if they were able to target and follow the frontal regions. In the Eastern basin (right panels), the improvement of the assimilation with MEDARGO floats is better in the middle and lower layers with respect to the surface, as already noticed in Sect. 4.1, due to high gradients present at the surface in regions where no data are present (Aegean Sea). For the XCTDs on the VOS tracks, data are collected also in the Aegean Sea (track 6 in Fig. 7), and the error is indeed lower in L1. 
T WEST WINTER
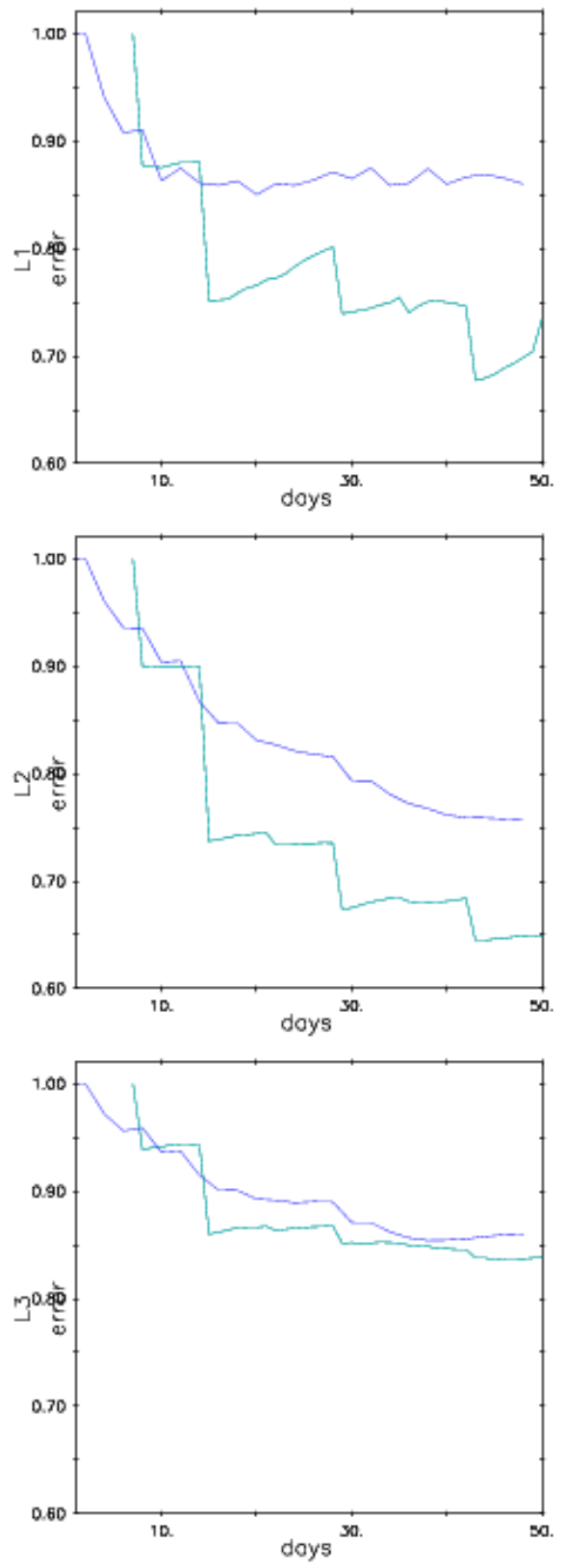

T EAST WINTER
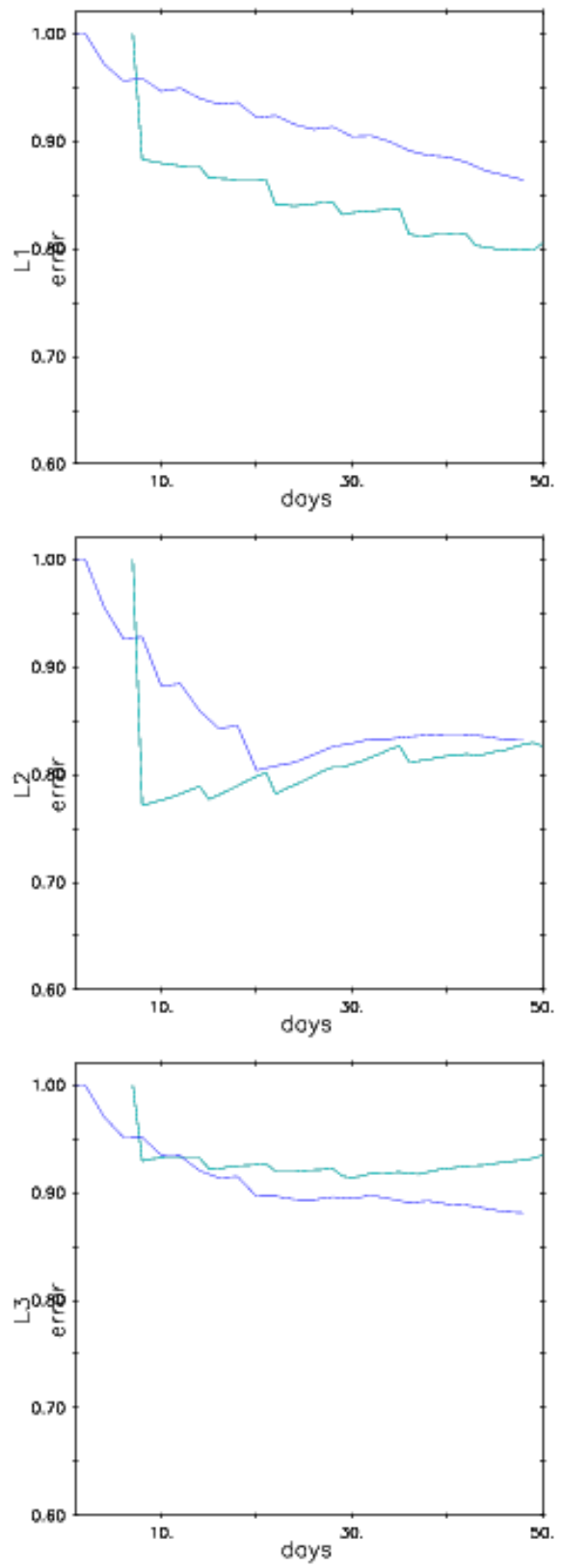

Fig. 10. Temperature relative error versus time in days for the idealized sampling experiments: Comparison between MEDARGO float assimilation from 47_WINTER (blue), and XCTD assimilation along VOS tracks (cyan) for the three layers L1,L2 and L3. Left panels are relative to the Western basin, right panelsot the eastern basin.

In conclusion, the results of the two experiments appear relatively similar, with the VOS data leading to a maximum improvement of approximately $10 \%$ with respect to MEDARGO. This is almost surprising given that the assimilated VOS profiles are about three times as many as the MEDARGO profiles, and it suggests that spatially sparser profiles from floats can be more efficient than fixed point profiles, probably because they approximately follow flow features. Also, it is possible that the VOS horizontal resolution of 12 nautical miles is redundant, at least in our OSSE experiments, in the sense that adjacent profiles might not be independent. This though could be a consequence of the MOM model resolution which does not completely resolve the mesoscale features. Further testing to assess this point is planned using a higher resolution model and appropriate statistical testing to verify data independence. Finally, it should 
be noticed that, even though in these idealized VOS experiments Salinity has been assimilated, expendable CTDs are not yet easily available for routine operation, and Temperature is mostly the only observable variable. This could represent a further argument for the advantage of MEDARGO floats observations, which are currently able to give both $\mathrm{T}$ and $\mathrm{S}$ profiles.

\section{Summary and conclusions}

OSSE experiments have been performed to investigate the impact of assimilating TS profiles from MEDARGO floats with different sampling strategies. Two sets of experiments consider the case of 47 floats over the whole Mediterranean Sea, approximately double the number in the present MFSTEP plan, launched along VOS tracks in the winter and summer season respectively. A third experiment considers the actual launching sites used during the MFSTEP 2004 summer releases, accounting for a total of 10 floats in the Western Mediterranean Sea.

Results for the experiments with 47 floats show that the TS assimilation is effective, with error reductions up to 20 $30 \%$. The most effective trajectories are the ones sampling frontal regions dividing different water masses, since even small differences in the mesoscale structure of the fronts can account for a significant portion of the error. For the intermediate layer L2, the most prominent and permanent fronts are observed in the Ionian Sea close to the Otranto Strait (dividing the LIW from the Adriatic water) and West of the Sardinia Channel (dividing the modified LIW from the water of Atlantic origin). Trajectories sampling these regions are launched along the Ionian track, track 5, and along the crossAlgerian basin track, track 2, respectively. For the surface layer L1, a front similar to the one in L2 is observed West of the Sardinia Channel, while the Ionian Sea front is absent. Less permanent and less well defined fronts are observed in the Levantin basin and near the coast, leading to a smaller error reduction in L1 with respect to L2, especially in summer. The trajectories sampling the Levantine basin fronts originate from along the eastern leg of track 1.

Results for the experiment with 10 floats launched at the realistic MFSTEP launching sites show a reduced impact of the assimilation. In some cases, the error can actually increase, at least temporarily, due to events where local corrections propagate dynamically, affecting regions that are not sufficiently sampled. If these regions are highly inhomogeneous with eddy anomalies of different signs, the correction propagation can result in corrections of the wrong sign and therefore in an error increase.

The OSSE results for float assimilation are in good agreement with results obtained for TS assimilation at fixed points along VOS tracks (as for XBT, Raicich, 2006). When considering an approximately double sampling with respect to the actual XBT MFSTEP plan, a VOS track assimilation is found to be effective with error reduction of $20-35 \%$. Consistently with the MEDARGO experiments with 47 floats, track 2 turns out to be highly effective in the western basin (the most effective is the very long track 1, but MEDARGO are not deployed along this track), and track 5 is the most effective in the eastern basin. Notice that the VOS experiments have a significantly higher horizontal resolution along tracks, resulting in a higher number of total profiles assimilated per week (approximately three times the MEDARGO floats). This suggests that profiles from floats, even though sparser, can be particularly effective, probably because they approximately follow flow features

In summary, the OSSE results show that frontal regions play a major role, as can be expected, and that it is crucial to maintain a sufficient coverage of them. A higher sampling than in the present MFSTEP is desirable, but if the number of floats cannot be increased, it might be better at least to concentrate them in the frontal areas. These results should be considered as useful suggestions, even though of course they are only indicative of the whole prediction problem. The OSSE experiments presented here, in fact, focus on errors related to incomplete knowledge of the initial state of the ocean, and are targeted to forecasting mesoscale anomaly structures. Possible biases in the solutions, model errors, incomplete knowledge of parameterizations and forcing are not considered here, and they might play an important role in practical applications. Also the sensitivity to the parameter values used in the assimilation scheme has not been considered. The same values as in the MFSTEP operational code have been maintained, and they might not be optimal since for instance the spatial scales are assumed to be constant while they are likely to depend on the specific region and on the season.

We conclude with a discussion on the potential and future perspectives of assimilating data from MEDARGO floats. We recall that, even though the present paper is focused on TS profile data, Argo floats provide also information on positions, which are linked to drift and therefore to velocity. While TS information are presently routinely assimilated in operational systems, position assimilation is still under experimentation and testing. A method for position assimilation have been recently developed (Molcard et al., 2005; Taillandier et al., 2006a) and implemented in a realistic Mediterranean Sea model (Taillandier et al., 2006b; Taillandier and Griffa, 2006). Even though the setting is different from the one considered here, a qualitative comparison can be drawn between twin experiment results for TS and position assimilation with similar data coverage. Results appear compatible, with similar values for the global error, even though at a more detailed level, the assimilation of positions seems to be more effective than the TS assimilation, especially for low (realistic) coverage. The correction in fact appears more consistent, and even though error oscillations due to correction propagation are occasionally present, they appear significantly reduced with respect to TS assimilation. This might be due 
to the fact that position assimilation leads to velocity correction, which is conceptually related (through geostrophy) to TS gradients. So, a conceptually higher order correction is introduced. Also, and more specifically, the method developed in Molcard et al. (2005) and Taillandier et al. (2006a, b) implies corrections of the velocity field balanced by corrections of the mass field, i.e. of TS. This correction is expected to provide more dynamically balanced results than the correction of TS only. In contrast the TS assimilation performed here is used only to update the density, without balancing geostrophically the velocity, and this can lead to sub-optimal solutions as shown by Burgers et al. (2002).

Regarding regions of most effective sampling, both types of assimilations indicate that the most effective floats are in regions of maximum variability, since the correction there is expected to be the highest. Unfortunately, regions of high velocity variability do not always correspond to strong gradients in TS, or vice versa. It would appear then that there could be a conflict in setting up a best sampling criteria, since it could depend on the specific variable which is considered. On the other hand, it should be considered that the error measurement we have used is restricted, since it is integrated and only reflects the size of the correction of a specific variable. It is likely that correcting the TS distribution in regions where it does not greatly contribute to the TS metric, might nevertheless significantly contribute to the correction of the other state variables like velocity and vice versa.

In this sense, an important recommendation for future work is that the assimilation of TS and position information should be done simultaneously using a multivariate approach in order to effectively extract all the possible dynamical information. This is expected to be especially helpful in the case of small scale structures (whose curvature is only partially resolved by the position information) with a well defined water mass signature, or in case of energetic boundary current with a reduced TS signal. Strong frontal areas are expected to contribute to both TS and velocity corrections, therefore providing especially effective sampling regions.

Acknowledgements. This work was supported by the European Commission (V Framework Program -Energy, Environment, and Sustainable Development) as part of the MFSTEP Project (contract number EVK3-CT-2002-00075).

Edited by: J. A. Johnson

\section{References}

Arnold Jr., C. P. and Dey, C. H.: Observing-system simulation experiments: Past, present and future, Bull. Am. Meteorol. Soc., 67, 687-695, 1986.

Barth, N. and Wunsch, C.: Oceanographic experiment design by simulated annealing, J. Phys. Oceanogr., 20, 1249-1263, 1990.

Bennett, A.F.: Inverse methods for assessing ship-of-opportunity networks and estimating circulation and winds from tropical ex- pendable bathythermograph data, J. Geophys. Res., 95, $16111-$ $16148,1990$.

Blanke, B., Arhan, M., Madec, G., and Roche, S.: Warm water paths in the Equatorial Atlantic as diagnosed with a General Circulation Model, J. Phys. Oceanogr. 29, 2753-2768, 1999.

Brasseur, P., Beckers, J. M., Brankart, J. M., and Schoenauen, R.: Seasonal temperature and salinity fields in the Mediterranean Sea: Climatological analyses of an historical data set, Deep-Sea Res., 43, 159-192, 1996.

Burgers, G., Balmaseda, M. A., Vossepoel, F. C., von Oldenborgh, G. J., and van Leeuwen, P. J.: Balanced ocean data assimilation near the Equator. J. Phys. Oceanogr., 32, 2509-2519, 2002.

De Mey, P.: Optimal interpolation in a model of the Azores current in 1986-88, in: Data assimilation: Tools for modelling the ocean in a global perspective, NATO/ASI Series, I/19, edited by: Brasseur, P. P. and Nihoul, J. C. J., Springer-Verlag, 253 pp, 1994.

De Mey, P.: Data assimilation at the oceanic mesoscale: A Review, J. Met. Soc. Japan, 75, Special issue on "Data assimilation in meteorology and oceanography: Theory and practice", 415-427, 1997.

De Mey, P. and Benkiran, M.: A multivariate reduced-order optimal interpolation method and its application to the Mediterranean basin-scale circulation, in: Ocean Forecasting Conceptual Basis and Applications, edited by: Pinardi, N. and Woods, J., Springer-Verlag, 281-306, 2002.

Demirov, E., Pinardi, N., Fratianni, C., Tonani, M., Giacomelli, L., and De Mey, P.: Assimilation scheme of Mediterranean Forecasting System: Operational implementation, Ann. Geophys., 21, 189-204, 2003, http://www.ann-geophys.net/21/189/2003/.

Fichaut, M., Balopoulos, E., Dooley, H., Garca-Fernandez, M.-J., Iona, A., Jourdan, D., Baudet, L., and Maillard, C.: A common protocol to assemble a coherent database from distributed heterogeneous data sets: The MEDATLAS database experience, In: Marine science and technology programme: Experiences in project data management, (Ed) Bohle-Carbonell, M., European Commission, Luxembourg, 349 pp, 1998.

Hackert, E. C., Miller R. N., and Busalacchi, A. J.: An optimized design for a moored instrument array in the tropical Atlantic Ocean, J. Geophys. Res., 103, 7491-7509, 1998.

Hernandez, F., Le Traon, P.-Y., and Barth, N. H.: Optimizing a drifter cast strategy with a genetic algorithm, J. Atmos. Ocean. Tech., 12, 330-345, 1994.

Kindle, J. C.: Sampling strategies and model assimilation of altimetric data for ocean monitoring and prediction, J. Geophys. Res., 91, 2418-2432, 1986.

Korres, G., Pinardi, N., and Lascaratos, A.: The ocean response to low-frequency interannual atmospheric variability in the Mediterranean Sea. Part I: sensitivity experiments and energy analysis, J. Climate, 13, 705-731, 2000.

Manzella, G. M. R., Scoccimarro, E., Pinardi, N., and Tonani, M.: Improved near-real time management procedures for the Mediterranean ocean Forecasting System - Voluntary Observing Ship program, Ann. Geophys., 21, 49-62, 2003, http://www.ann-geophys.net/21/49/2003/.

Miyakoda, K., Smagorinsky, J., Strickler, R. F., and Hembree, G. D.: Experimental extended predictions with a nine-level hemispheric model, Mon. Wea. Rev., 97, 1-76, 1969. 
Molcard, A., Griffam A., and Ozgokmenm T.: Lagrangian data assimilation in multilayer primitive equation models, J. Atmos. Oceanic Technol., 22, 70-83, 2005.

Pacanowski, R. C., Dixon, K., and Rosati, A.: The GFDL Modular Ocean Model users guide, version 1.0, Geophysical Fluid Dynamics Laboratory Ocean Tech. Rep., 2, 18 pp., 1990.

Poulain, P.-M.: A profiling float program in the Mediterranean, Argonautics, 6, 2, 2005.

Raicich, F. and Rampazzo, A.: Observing System Simulation Experiments for the assessment of temperature sampling strategies in the Mediterranean Sea, Ann. Geophys., 21, 151-165, 2003, http://www.ann-geophys.net/21/151/2003/.

Raicich, F.: The assessment of temperature and salinity sampling strategies in the Mediterranean Sea: Idealized and real cases, Ocean Sci., 2, 97-112, 2006,

http://www.ocean-sci.net/2/97/2006/.

Rohaly, G. D. and Krishnamurti, I. N.: An observing system simulation experiment for the Laser Atmospheric Wind Sounder (LAWS), J. Appl. Meteorol., 32, 1453-1471, 1993.

She J., Høyer, J. L., and Larsen, J.: Assessment of sea surface temperature observational networks in the Baltic Sea and North Sea. J. Mar. Sys, Special Issue "Marine Environmental Monitoring and Prediction", in press, 2006.
Sparnocchia, S., Pinardi, N., and Demirov, E.: Multivariate Empirical Orthogonal Function analysis of the upper thermocline structure of the Mediterranean Sea from observations and model simulations, Ann. Geophys., 21, 167-187, 2003, http://www.ann-geophys.net/21/167/2003/.

Taillandier, V., Griffa, A., and Molcard, A.: A variational approach for the reconstruction of regional scale Eulerian velocity fields from Lagrangian data, Ocean Modell., 13, 1-24, 2006 a.

Taillandier V., Griffa, A., Poulain, P.-M., and Béranger, K.: Assimilation of "Argo" float positions in the North Western Mediterranean Sea and impact on ocean circulation simulations, Geophys. Res. Lett., 33, L11604, doi:10.1029/2005GL025552, $2006 b$.

Taillandier, V. and Griffa, A.: Implementation of position assimilation for Argo floats in a realistic Mediterranean Sea OPA model and twin experiment testing, Ocean Sci., 2, 223-236, 2006, http://www.ocean-sci.net/2/223/2006/. 